SHORT REPORT

\title{
Can an information booklet on an ethnic minority increase the knowledge base of junior doctors?
}

\author{
B Ward, P de Chazal, J F Mayberry
}

See end of article for

authors' affiliations

Postgrad Med J 2004;80:219-220. doi: 10.1136/pgmi.2004.019802

Correspondence to:

Dr J F Mayberry, Leicester General Hospital,

Gwendolen Road

Leicester LE5 4PW, UK;

jmaybe@tiscali.co.uk

Submitted 8 May 2003

Accepted 17 June 2003
Many doctors encounter people of different cultural backgrounds for the first time as patients. In Leicester a significant proportion of the area's population comes from a Gujarati and Hindu background. In an attempt to better inform junior doctors about the views and beliefs of their patients, a group of clinicians and administrators developed an information booklet about the beliefs and practices of people from this community. The impact of this booklet on a group of 54 junior doctors' knowledge was investigated over a period of one month. Such an information booklet was found to significantly increase awareness of the cultural background of patients from a minority community and this knowledge was maintained for at least one month after distribution. The study did not investigate impact on attitudes.
M inority patients can experience problems during hospitalisation because of ignorance on the part of carers, including junior hospital doctors. There is a growing awareness of a need to address these issues and this can include information packs, cultural awareness days, and the provision of good quality information. An easy-to-read booklet was therefore professionally developed to provide basic information about the Gujarati population in Leicester. It was given to hospital doctors on their induction day and its effect on their knowledge levels tested one month later.

\section{METHODS}

Fifty four doctors of various ethnic backgrounds, who were either junior or senior house officer grade, were asked to take part in a study to evaluate an information booklet about Gujarati Hindus. ${ }^{1}$ The approach was made on their induction programme. The purpose and background to the booklet was described. It had been prepared as a result of a programme of research directed at the needs of South Asian patients living in Leicestershire and the perceptions of junior doctors about their lifestyle and experience of illness. These unpublished studies had identified areas in which junior doctors lacked knowledge and the booklet was developed as an educational and factual tool. It was not seen as a method of dealing with discrimination or prejudice, but rather as a source of information. During the induction it was clearly stated that doctors were not expected to "learn" its contents, but rather to use it as a reference work. All doctors were asked to complete an initial questionnaire which tested knowledge of religious festivals, dress, disease, and demography (Appendix). This was completed before distribution of the booklet. They were told that the questionnaire would be readministered one month later. They were encouraged to make reference to the booklet, if still available to them at that time, during the retest. The study was based on voluntary completion of the questionnaires and any non-responders would not be interviewed.

\section{RESULTS}

All 54 doctors agreed to participate in the study and completed the initial questionnaire before distribution of the booklet. They had received no formal instruction about patients from the Gujarati community. They were given a test which consisted of six questions (Appendix). Each correct answer was scored as 1, with no deductions for wrong answers. Their mean (SD) score was $3.3(0.8)$.

One month later, an identical questionnaire was readministered. Retrospective analysis was conducted by dividing the participants into two subgroups. The first subgroup (subgroup A) comprised 32 (59\%) doctors who completed only the initial questionnaire. The second, subgroup B, comprised $22(41 \%)$ doctors who completed both the initial and subsequent questionnaires.

Analysis of the mean (SD) scores for the initial questionnaire showed that those in subgroup A had a similar score $(3.5(0.7))$ to those in subgroup B (3.1 (0.9)) with no statistically significant difference. However, the mean score of subgroup B rose significantly to 4.6 (1.1) when these participants repeated the questionnaire one month later (paired $t$ test $=-5.9, \mathrm{p}<0.0001$ ) (table 1 ).

The scores obtained for individual questions were compared by subgroup using a z statistic. ${ }^{2}$ A significant difference existed in the scores for question 5, which dealt with illness experience. Those in subgroup B did significantly less well on the initial questionnaire than those in subgroup A. No other significant differences were detected.

After readministration of the questionnaire, the scores for individual questions were compared, again using a z statistic. Questions 2, 3, 4 and 6, which dealt with aspects of Gujarati life in the United Kingdom, all showed significant improvement (table 2).

\section{CONCLUSIONS}

Our study has shown that an easy-to-read professionally produced booklet about minority communities can lead to sustained greater knowledge among junior doctors. Whether

Table 1 Comparison of mean (SD) test scores (range 06) before and after distribution of booklet

\begin{tabular}{lll}
\hline & $\begin{array}{l}\text { Score for initial } \\
\text { questionnaire before } \\
\text { distribution }\end{array}$ & $\begin{array}{l}\text { Score on readministering } \\
\text { identical questionnaire } \\
\text { after distribution }\end{array}$ \\
\hline $\begin{array}{l}\text { Subgroup A } \\
(\mathrm{n}=32)\end{array}$ & $3.5(0.7)$ & Failed to complete \\
$\begin{array}{l}\text { Subgroup B } \\
(\mathrm{n}=22)\end{array}$ & $3.1(0.9)$ & $4.6(1.1)$ \\
\hline
\end{tabular}




\begin{tabular}{|c|c|c|c|c|c|}
\hline \multirow[b]{2}{*}{ Question No } & \multicolumn{2}{|l|}{ Before distribution } & \multicolumn{2}{|l|}{ After distribution } & \multirow[b]{2}{*}{$\mathrm{p}$ Value } \\
\hline & Subgroup $A(n=32)$ & Subgroup B ( $n=22$ ) & Subgroup $B(n=22$ ) & $z$ & \\
\hline 1 & 31 & 22 & 22 & & NS \\
\hline 2 & 19 & 12 & 18 & -1.9 & $<0.05$ \\
\hline 3 & 6 & 3 & 10 & -1.9 & $<0.05$ \\
\hline 4 & 25 & 15 & 21 & -2.3 & $<0.02$ \\
\hline 5 & $31^{*}$ & $15^{*}$ & 19 & & NS \\
\hline 6 & 0 & 0 & 11 & -3.8 & $<0.0001$ \\
\hline
\end{tabular}

this will affect personal attitudes or clinical practice must await further research. However, there is a view that when knowledge replaces ignorance discriminatory practices are reduced in frequency.

In 1986 Poulton et al reviewed the training of medical students and aspects of the practice of medicine in a multiracial and multicultural Britain. ${ }^{3}$ They found it had a low priority and was frequently absent from the curriculum. However, junior doctors, clinical tutors, and administrators acknowledged the need for formal training. The more general need for a staff training programme about the needs of ethnic minority patients was again confirmed in a case control study of 52 Asian patients in Middlesborough in 1992. Although this study dealt with the practical day-to-day requirements of patients, it emphasised the need for educational material and other information about the culture of minority groups. ${ }^{4}$

However, we should not become complacent. A study from Arizona has suggested that limited training about various minorities may simply make health carers more aware of their inadequacies in providing culturally sensitive care. ${ }^{5}$ The development of information booklets should be simply the first step in a programme of on-going education about the local communities which any hospital serves.

\section{Authors' affiliations}

B Ward, P de Chazal, J F Mayberry, Leicester General Hospital, Gwendolen Road, Leicester, UK

\section{APPENDIX}

\section{QUESTIONNAIRE ON GUJARATI HINDUS}

\section{Name:......}

Department:....

Extension:

Please circle correct answer

1. Which of the following celebrations is the main Hindu celebration of the triumph of light over darkness:

- A. Diwali

- B. Eid

- C. Easter

2. Which of the following towns has a large Gujarati population:

- A. Glasgow

- B. Cardiff

- C. Bolton
3. English is spoken at home by what percentage of Gujarati families:

- A. $10 \%$

- B. $25 \%$

- C. $40 \%$

- D. $65 \%$

- E. $90 \%$

4. A bindi is:

- A. A special form of Indian dress

- B. A meal

- C. A red spot worn on the forehead

- D. A religious ceremony

5. Which of the following illnesses are uncommon in Gujarati people:

- A. Heart disease

- B. Diabetes

- C. Cancer

6. Indians were first elected to the British Parliament in the:

- A. 1890 s

- B. 1920s

- C. $1940 \mathrm{~s}$

- D. $1960 \mathrm{~s}$

- E. $1980 \mathrm{~s}$

Thank you for completing this questionnaire.

\section{CORRECT ANSWERS}

1, A; 2, C; 3, C; 4, C; 5, C; 6, A.

\section{REFERENCES}

1 Mayberry JF, de Chazal P. Gujarati Hindus. A guide for health and public service workers. Leicester: Leicester General Hospital (undated).

2 Fleiss J. Statistical methods for rates and proportions. 2nd Ed. Chichester: Wiley, 1981.

3 Poulton J, Rylance GW, Johnson MR. Medical teaching of the cultural aspects of ethnic minorities: does it exist? Med Educ 1986;20:492-7.

4 Madhok R, Bhopal RS, Ramaiah RS. Quality of hospital service: a study comparing Asian and "non-Asian" patients in Middlesborough. J Public Health Med 1992;14:271-9.

5 Alpers RR, Zoucha R. Comparison of cultural competency and cultural confidence of senior nursing students in a private southern university. Journal of Cultural Diversity 1996;3:9-15. 